\title{
Robust Tracking Control for Robotic Manipulator via Fuzzy Logic System and $H_{\infty}$ Approaches
}

\author{
Kun Mu, ${ }^{1}$ Cong Liu, ${ }^{2}$ and Jinzhu Peng ${ }^{3,4}$ \\ ${ }^{1}$ Department of Computer Science and Engineering, Henan Institute of Engineering, Zhengzhou, Henan 451191, China \\ ${ }^{2}$ College of Information \& Business, Zhongyuan University of Technology, Zhengzhou, Henan 450001, China \\ ${ }^{3}$ School of Electrical Engineering, Zhengzhou University, Zhengzhou, Henan 450007, China \\ ${ }^{4}$ Henan Senyuan Electric Co. Ltd., Zhengzhou, Henan 450047, China
}

Correspondence should be addressed to Jinzhu Peng; jzpeng@zzu.edu.cn

Received 8 January 2015; Revised 17 March 2015; Accepted 17 March 2015

Academic Editor: James Lam

Copyright (C) 2015 Kun Mu et al. This is an open access article distributed under the Creative Commons Attribution License, which permits unrestricted use, distribution, and reproduction in any medium, provided the original work is properly cited.

\begin{abstract}
Based on fuzzy logic system (FLS) and $H_{\infty}$ control methodologies, a robust tracking control scheme is proposed for robotic system with uncertainties and external disturbances. FLS is employed to implement the framework of computed torque control (CTC) method via its approximate capability which is used to attenuate the nonlinearity of robotic manipulator. The robust $H_{\infty}$ control can guarantee robustness to parametric and dynamics uncertainties and also attenuate the effect of immeasurable external disturbances entering the system. Moreover, a quadratic stability approach is used to reduce the conservatism of the conventional robust control approach. It can be guaranteed that all signals in the closed-loop are bounded by employing the proposed robust tracking control. The validity of the proposed control scheme is shown by simulation of a two-link robotic manipulator.
\end{abstract}

\section{Introduction}

Tracking control of robotic manipulator is always a challenging problem in control fields due to its uncertainties, disturbances, and nonlinear dynamics $[1,2]$. Over the past decades, various control approaches have been applied for controlling the challenging robotic manipulator systems, such as PID control [3], computed torque control (CTC) method [4-6], variable structure control [7-9], fuzzy control [5, 10-12], and neural networks control [13-18].

It is noteworthy that varieties of hybrid control systems have been designed for controlling the complex robotic systems using different combinations of the above-mentioned methods. For example, in $[13,15]$, neural networks were utilized to approximate the equivalent control of variable structure control entirely, and then the $H_{\infty}$ techniques were applied to achieve certain tracking performance. As a feedback linearization control technique, CTC was usually included in controller designing for complex robotic system due to its good performances $[5,6,16,17]$. In order to eliminate the effect of the uncertainties, Song et al. [5] proposed an approach of CTC plus fuzzy compensator for robotic system; the nominal system was controlled by using CTC method and for uncertain system a fuzzy controller acts as compensator. In [16], CTC plus a neural network compensator was proposed and simulations were conducted on a two-link robotic manipulator; furthermore, an experimental example was tested on PUMA560. However, they assumed that system actual acceleration was measurable. Although the acceleration can be obtained through installing accelerometers on the robotic systems, the measurement noises and weight of these extra utilities would both sacrifice the tracking performance of robotic systems [17]. Without robotic system acceleration information, Peng et al. [17] proposed robust hybrid tracking control for robotic system, which combines CTC with a neural network-based robust compensator. Kim et al. [19] presented a model-based motion control approach for industrial robots by considering a serial two-link robot arm model with joint nonlinearities. Chen et al. [20] proposed two types of adaptive control scheme combining conventional CTC and different fuzzy compensators for the robust tracking control of robotic manipulators with structured and unstructured uncertainties. Recently, Peng and Liu [6] proposed an adaptive robust quadratic stabilization tracking control scheme 
that combines CTC, nonlinear $H_{\infty}$ control, and variable structure control for robotic manipulator. Peng et al. [21] proposed a fuzzy adaptive output feedback control scheme based on fuzzy adaptive observer for robotic systems with parameter uncertainties and external disturbances. It should be pointed out that, in the above controllers, the nominal model of robotic manipulator was assumed to be known exactly. However, in practical engineering, this assumption may be unreasonable due to the nonlinear functions that exist in the nominal model.

Compared with the similar control methods that were proposed by the above and existing literatures, the nominal model of robotic manipulator, in this paper, is no longer needed to be completely known, whereas only the nominal inertia matrix is assumed to be known. A robust tracking control scheme, which combines FLS and robust $H_{\infty}$ control for robotic manipulator, is then proposed. In the proposed scheme, FLS is employed to implement the framework of CTC method via its approximate capability which is used to attenuate the nonlinearity of robotic manipulator. The robust $H_{\infty}$ control approach can guarantee robustness to parametric and dynamics uncertainties. It can be guaranteed that all signals in the closed-loop are bounded by employing the proposed FLS-based robust tracking control. The validity of the control scheme is shown by computer simulation of a twolink robotic manipulator.

This paper is organized as follows. In Section 2, the description of a fuzzy logic system is included, and some preliminaries are addressed, which consist of mathematical notations, dynamical models of robotic manipulators with uncertainties, and detailed explanation related to CTC for robotic manipulators. The design of robust tracking control based on FLS is given in Section 3, and the robust stability is analyzed. The MATLAB simulation results are given in Section 4, and the conclusions are drawn in Section 5.

\section{Preliminaries}

Standard notations are used in this paper. Let $\mathfrak{R}$ be the real number set, let $\Re^{n}$ be the $n$-dimensional vector space, and let $\mathfrak{R}^{n \times n}$ be the $n \times n$ real matrix space. The norm of vector $x \in \mathfrak{R}^{n}$ and that of matrix $A \in \mathfrak{R}^{n}$ are defined, respectively, as $\|x\|=\sqrt{x^{T} x}$ and $\|A\|=\operatorname{tr}\left(A^{T} A\right)$. If $y$ is a scalar, then $\|y\|$ denotes the absolute value. $\lambda_{\min }(A)$ and $\lambda_{\max }(A)$ are the minimum and the maximum eigenvalues of matrix $A$, respectively. $I_{n} \in \mathfrak{R}^{n \times n}$ is an $n \times n$ identity matrix, $\operatorname{tr}(\cdot)$ is the trace operator, and $\operatorname{sgn}(\cdot)$ is the standard sign function.

2.1. Description of Fuzzy Logic System. As shown in Figure 1, a typical fuzzy system comprises the fuzzy rule base, the fuzzifier, the fuzzy inference engine working on fuzzy rules, and the defuzzifier. The fuzzy inference engine performs a mapping from fuzzy sets in input space $U \in \mathfrak{R}^{n}$ to fuzzy sets in output space $V \in \mathfrak{R}^{m}$ based on fuzzy rules, where

$$
\begin{aligned}
& U=U_{1} \times U_{2} \times \cdots \times U_{n}, \quad U_{i} \in \mathfrak{R} \text { for } i=1,2, \ldots, n, \\
& V=V_{1} \times V_{2} \times \cdots \times V_{m}, \quad V_{j} \in \mathfrak{R} \text { for } j=1,2, \ldots, m .
\end{aligned}
$$

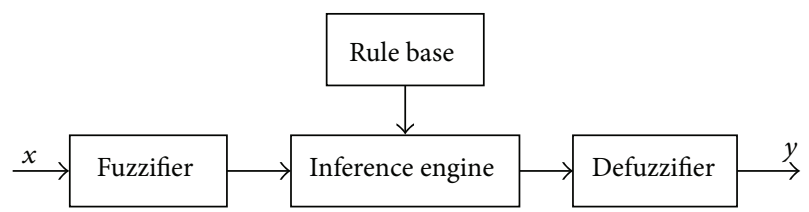

Figure 1: The basic configuration of a fuzzy logic system.

In general, consider a system with $n$ inputs $x=\left[x_{1}, x_{2}\right.$, $\left.\ldots, x_{n}\right]^{T}$ and the output data $y=\left[y_{1}, y_{2}, \ldots, y_{m}\right]^{T}$; the $i$ th fuzzy rule has the following form.

$R^{i}$ : If $x_{1}$ is $A_{1}^{i}$ and $\ldots$ and $x_{n}$ is $A_{n}^{i}$ then $y_{1}$ is $B_{1}^{i}$ and $\ldots$ and $y_{m}$ is $B_{m}^{i}, i=1,2, \ldots, M$, where $i$ is the number of fuzzy rules, $M$ is the total number of rules, and $A_{p}^{i}$ and $B_{q}^{i}(p=1, \ldots, n, q=1, \ldots, m)$ are the fuzzy sets of the antecedent part and the real numbers of the consequent part, described by their membership functions $\mu_{A_{p}^{i}}\left(x_{p}\right)$ and $\mu_{B_{q}^{i}}\left(y_{q}\right)$, respectively.

Once the inputs $x=\left[x_{1}, x_{2}, \ldots, x_{n}\right]^{T}$ are given, the output $y$ of the fuzzy inference system with weighted-center defuzzifier, product inference, and singleton fuzzifier can be derived from the following form:

$$
y(x)=\frac{\sum_{i=1}^{M} \bar{y}^{i}\left(\prod_{p=1}^{n} \mu_{A_{p}^{i}}\left(x_{p}\right)\right)}{\sum_{i=1}^{M}\left(\prod_{p=1}^{n} \mu_{A_{p}^{i}}\left(x_{p}\right)\right)},
$$

where $\bar{y}^{i}$ is the point in $V_{j}$ at which $\mu_{B_{q}^{i}}\left(\bar{y}^{i}\right)$ achieves its maximum value. Without loss of generality, we assume that $\mu_{B_{q}^{i}}\left(\bar{y}^{i}\right)=1$.

Assume that fuzzy basis functions are defined as follows:

$$
\phi_{i}(x)=\frac{\prod_{p=1}^{n} \mu_{A_{p}^{i}}\left(x_{p}\right)}{\sum_{i=1}^{M}\left(\prod_{p=1}^{n} \mu_{A_{p}^{i}}\left(x_{p}\right)\right)} .
$$

Then, the fuzzy system in (2) can be rewritten as

$$
y(x)=\sum_{i=1}^{M} \bar{y}^{i} \phi_{i}(x)=\theta^{T} \phi(x),
$$

where $\phi(x)=\left[\phi_{1}(x), \phi_{2}(x), \ldots, \phi_{m}(x)\right]^{T} \in \mathfrak{R}^{M}$ is called the fuzzy basic function vector or the antecedent function vector and $\theta=\left[\bar{y}^{1}, \bar{y}^{2}, \ldots, \bar{y}^{i}\right]^{T} \in \Re^{M}$ is called the parameter vector.

It has been proved that the adaptive fuzzy system can approximate any real continuous function over a compact set to arbitrary accuracy [10].

2.2. Robotic Manipulator Dynamic. Consider a general $n$ link rigid robotic manipulator, which takes into account the external disturbances, with the equation of motion given by $[5,17]$

$$
M(q) \ddot{q}+C(q, \dot{q}) \dot{q}+G(q)+\tau_{d}=\tau,
$$

where $q$, $\dot{q}$, and $\ddot{q} \in \mathfrak{R}^{n}$ are joint position, velocity, and acceleration vectors of the robot, respectively; $M(q) \in \mathfrak{R}^{n \times n}$ is 
the symmetric and positive definite inertia matrix; $C(q, \dot{q}) \in$ $\mathfrak{R}^{n \times n}$ is the effect of Coriolis and centrifugal forces; $G(q) \in$ $\mathfrak{R}^{n}$ is the gravity vector; $\tau_{d} \in \mathfrak{R}^{n}$ denotes unknown disturbances including unstructured dynamics and unknown payload dynamics. $\tau \in \mathfrak{R}^{n}$ is the torque input vector.

For convenience, dynamical model (5) can be rewritten as the following compact form:

$$
M(q) \ddot{q}+H(q, \dot{q})+\tau_{d}=\tau,
$$

where $H(q, \dot{q})=C(q, \dot{q}) \dot{q}+G(q)$.

The following properties are required for the subsequent development.

Property 1. The inertia matrix $M(q)$ is symmetric and positive definite, which is uniformly bounded and satisfies

$$
m_{m} I_{n} \leq M(q) \leq m_{M} I_{n}, \quad \forall q \in \mathfrak{R}^{n},
$$

where $m_{m}$ and $m_{M}$ are some positive constants.

Property 2. The matrix $\{\dot{M}(q)-2 C(q, \dot{q})\}$ is skew-symmetric; that is,

$$
x^{T}\{\dot{M}(q)-2 C(q, \dot{q})\} x=0, \quad \forall x \in \mathfrak{R}^{n} .
$$

Assumption 1. Disturbance is bounded by $\left\|\tau_{d}\right\| \leqslant \tau_{D}$, where $\tau_{D}$ is some positive constant.

The parameters $M(q), C(q, \dot{q})$, and $G(q)$ in dynamical model (5) are functions of physical parameters of manipulators like links masses, links lengths, moments of inertia, and so on. The precise values of these parameters are difficult to acquire due to measuring errors, environment, and payloads variations. Therefore, here it is assumed that actual values $M(q), C(q, \dot{q})$, and $G(q)$ can be separated as nominal parts denoted by $M_{0}(q), C_{0}(q, \dot{q})$, and $G_{0}(q)$ and uncertain parts denoted by $\Delta M(q), \Delta C(q, \dot{q})$, and $\Delta G(q)$, respectively. These variables satisfy the following relationships:

$$
\begin{gathered}
M(q)=M_{0}(q)+\Delta M(q), \\
C(q, \dot{q})=C_{0}(q, \dot{q})+\Delta C(q, \dot{q}), \\
G(q)=G_{0}(q)+\Delta G(q) .
\end{gathered}
$$

Assumption 2. The bound of uncertainty parameters is known, which can be expressed as

$$
\|\Delta M(q)\| \leqslant \delta_{M}, \quad\|\Delta C(q, \dot{q})\| \leqslant \delta_{C}, \quad\|\Delta G(q)\| \leqslant \delta_{G},
$$

where $\delta_{M}, \delta_{C}$, and $\delta_{G}$ are positive constants.

Suppose that dynamical models of robotic manipulators are known precisely and unmodeled dynamics are excluded; that is, $\Delta M(q), \Delta C(q, \dot{q}), \Delta G(q)$, and $\tau_{d}$ are all zeros. At this time, dynamical models (6) can be converted into the following nominal model:

$$
M_{0}(q) \ddot{q}+H_{0}(q, \dot{q})=\tau,
$$

where $H_{0}(q, \dot{q})=C_{0}(q, \dot{q}) \dot{q}+G_{0}(q)$.
2.3. Computed Torque Control Method for Robot. As a nonlinear feedback linearization approach, CTC method is a common control scheme for nonlinear system; in particular, there are strongly coupled and highly nonlinear manipulator dynamics. According to the CTC method, the control law for robot can be chosen as

$$
\tau=M_{0}(q)\left(\ddot{q}_{d}+K_{v} \dot{e}+K_{p} e\right)+H_{0}(q, \dot{q}),
$$

where $e$ are the tracking error defined by $e=q_{d}-q$ and $q$ and $q_{d}$ are the joint and desired output trajectories for each joint. The coefficients $K_{v}$ and $K_{p}$ should be chosen such that all the roots of the polynomial $h(s)=s^{2}+K_{v} s+K_{p}$ are in the open left-half plane.

Assumption 3. The desired trajectories $q_{d}$ are continuous and bounded known functions of time with bounded known derivatives up to the second order.

$$
\begin{aligned}
& \text { Substituting (12) into (11) yields } \\
& \qquad \ddot{e}+K_{v} \dot{e}+K_{p} e=0 .
\end{aligned}
$$

Remark 4. It should be noted that CTC approach is based on feedback linearization technique, which results in a linear time-invariant closed-loop system (13), implying acquirement of globally asymptotical stability. Furthermore, explicit conditions for performance indices may be obtained in terms of controller gain matrices. More specifically, globally asymptotical stability is guaranteed provided that $K_{p}$ and $K_{v}$ in (13) are symmetric and positive definite constant matrices.

According to analysis above, CTC strategy relies on strong assumptions that exact knowledge of robotic dynamics is precisely known and unmodeled dynamics has to be ignored, which is impossible in practical engineering. Motivated by the idea of CTC introduced above, one can image that CTC is used to control nominal system and another robust controller attaching to CTC for uncertain system can be designed. In this way, applying (12) and (13) to practical robotic manipulator system (6) yields

$$
\ddot{e}+K_{v} \dot{e}+K_{p} e=f(q, \dot{q})
$$

where $f(q, \dot{q})=M_{0}^{-1}(q)\left[\Delta M(q) \ddot{q}+\Delta C(q, \dot{q}) \dot{q}+\Delta G(q)+\tau_{d}\right]$ is a function of joint variables, physical parameters, parameters variations, unmodeled dynamics, and so on.

The controller can be defined as

$$
\tau=\tau_{0}-u,
$$

where $\tau_{0}$ is CTC defined like (12) and $u$ is a robust control law to be determined in next section.

Remark 5. All the parameters in the proposed scheme may be uncertain, which is true in practical situation. In some publications like [9], it is clearly unrealistic that only Coriolis terms, gravity terms, and friction terms may be uncertain, whereas inertia matrix was assumed to be known exactly. Literatures $[5,6,17]$ assumed that the nominal system is completely known, which is controlled using computed torque 
method. Aiming at the uncertain system, a fuzzy or neural controller acts as compensator for computed torque method. However, in practical engineering, this assumption may be unreasonable. Therefore, an assumption is given as follows. Then, we will utilize an FLS to approximate the nominal system in next section.

Assumption 6. It is assumed that only the nominal parameter of the inertia matrix $M_{0}(q)$ is known; other nominal parameters $C_{0}(q, \dot{q})$ and $G_{0}(q)$ are both assumed to be unknown.

Remark 7. Assumption 6 is reasonable, since in reality the inertia matrix of a robotic manipulator is relatively easy to obtain an acceptable precision; furthermore, the parametric uncertainty of inertia matrix is considered.

Up to now, the control objective can be restated to seek a robust control law as a compensator for CTC, which is approximated by FLS due to the unknown nominal parameters $C_{0}(q, \dot{q})$ and $G_{0}(q)$, such that joint motions of robotic systems (6) can follow the desired trajectories.

\section{Robust Tracking Control Design Based on FLS}

In this section, FLS is employed to implement the framework of CTC method (12). Firstly, it is assumed that the right-hand side in (12) can be represented by an ideal:

$$
\tau_{0}=M_{0}(q)\left(\ddot{q}_{d}+K_{v} \dot{e}+K_{p} e\right)+\theta^{T} \phi(q, \dot{q}),
$$

where $\theta^{T} \phi(q, \dot{q})$ is fuzzy system of the form of (4). $\theta$ is the weight matrix and $\phi(q, \dot{q})$ denotes generalization result. We assume that there exists $\Omega_{\theta}$, and ideal parameter is in the compact set $\Omega_{\theta}$. The ideal parameter can be defined as

$$
\theta^{*}=\arg \min _{\theta \in \Omega_{\theta}}\left\{\sup \mid H_{0}(q, \dot{q})-\theta^{T} \phi(q, \dot{q})\right\} \text {. }
$$

Assumption 8. The norm of optimal weight matrix $\theta^{*}$ is bounded so that $\left\|\theta^{*}\right\| \leq M_{\theta}$. The reconstruction error $\varepsilon$ is bounded; that is, $\|\varepsilon\| \leq \delta_{\varepsilon}$, where $\delta_{\varepsilon}$ is a positive constant.

Using control law equations (12) and (15), the closed-loop system becomes

$$
\begin{aligned}
M(q) \ddot{q}+C(q, \dot{q}) \dot{q}+G(q) \\
=M_{0}(q)\left(\ddot{q}_{d}+K_{v} \dot{e}+K_{p} e\right)+\theta^{T} \phi(q, \dot{q})-u-\tau_{d} \\
=M_{0}(q)\left(\ddot{q}_{d}+K_{v} \dot{e}+K_{p} e\right)+C_{0}(q, \dot{q}) \dot{q}+G_{0}(q) \\
\quad-\varepsilon-\widetilde{\theta}^{T} \phi(q, \dot{q})-u-\tau_{d}
\end{aligned}
$$

where $\widetilde{\theta}=\theta^{*}-\theta$ denoting error of weight matrix and $\varepsilon$ is a minimum reconstructed error vector. Then,

$$
\begin{aligned}
& M(q) \ddot{e}+\left[M_{0}(q) K_{v}+\Delta C(q, \dot{q})\right] \dot{e}+M_{0}(q) K_{p} e \\
& =u+\tau_{d}+\varepsilon+\widetilde{\theta}^{T} \phi(q, \dot{q})+\Delta M(q) \ddot{q}_{d} \\
& \quad+\Delta C(q, \dot{q}) \dot{q}_{d}+\Delta G(q) .
\end{aligned}
$$

We define the state vector as

$$
x=\left[\begin{array}{c}
x_{1}^{T} \\
x_{2}^{T}
\end{array}\right]=\left[\begin{array}{l}
e^{T} \\
\dot{e}^{T}
\end{array}\right] .
$$

The state-space equation has the form of

$$
\begin{gathered}
\dot{x}_{1}=x_{2}, \\
M(q) \dot{x}_{2}=u+\tau_{d}+\varepsilon+\widetilde{\theta}^{T} \phi(q, \dot{q})+\Delta M(q) \ddot{q}_{d} \\
+\Delta C(q, \dot{q}) \dot{q}_{d}+\Delta G(q) \\
-\left[M_{0}(q) K_{v}+\Delta C(q, \dot{q})\right] x_{2}-M_{0}(q) K_{p} x_{1} .
\end{gathered}
$$

Defining $\omega=\Delta M(q) \ddot{q}_{d}+\Delta C(q, \dot{q}) \dot{q}_{d}+\Delta G(q)$, the statespace equation has the form of

$$
\dot{x}=A x+B\left(u+\tau_{d}+\varepsilon+\widetilde{\theta}^{T} \phi(q, \dot{q})+\omega\right),
$$

where

$$
\begin{gathered}
A=A_{0}+\Delta A, \quad B=\left(B_{0}+\Delta B\right) M_{0}^{-1}(q), \\
A_{0}=\left[\begin{array}{cc}
0_{n \times n} & I_{n \times n} \\
-K_{p} & -K_{v}
\end{array}\right],
\end{gathered}
$$

$\Delta A$

$$
\begin{gathered}
=\left[\begin{array}{cc}
0_{n \times n} & I_{n \times n} \\
M^{-1}(q) \Delta M(q) K_{p} & M^{-1}(q)\left[\Delta M(q) K_{v}-\Delta C(q, \dot{q})\right]
\end{array}\right], \\
B_{0}=\left[\begin{array}{c}
0_{n \times n} \\
I_{n \times n}
\end{array}\right], \quad \Delta B=\left[\begin{array}{c}
0_{n \times n} \\
-M^{-1}(q) \Delta M(q)
\end{array}\right] .
\end{gathered}
$$

Assumption 9. $\Delta A$ and $\Delta B$ represent the time-varying parametric uncertainties having the following structure:

$$
\left[\begin{array}{ll}
\Delta A & \Delta B
\end{array}\right]=D F\left[\begin{array}{ll}
E_{a} & E_{b}
\end{array}\right],
$$

where $D, E_{a}$, and $E_{b}$ are known constant matrix appropriate dimensions, and $F \in \Re^{n \times n}$ is unknown Lebesgue measurable matrix which is bounded as follows:

$$
F^{T} F \leq I_{n \times n} .
$$

Lemma 10 (see [22]). Let $X, Y$, and $F$ be real matrices with appropriate dimensions and $F^{T} F \leq I_{n \times n}$. Then, for any scalar $\alpha>0$,

$$
X F Y+Y^{T} F^{T} X^{T} \leq \alpha X X^{T}+\alpha^{-1} Y^{T} Y .
$$

Theorem 11. Considering the dynamic equation (11), suppose that Assumptions 1, 2, 3, 6, 8, and 9 are satisfied, and $\tau_{d} \epsilon$ $L_{2}[0,+\infty)$. If there exist a matrix $P=P^{T}>0$ and positive numbers $\gamma, \eta$, such that the following matrix inequality holds

$$
\begin{aligned}
P A_{0} & +A_{0}^{T} P+\frac{1}{\rho^{2}} P \bar{B} \bar{B}^{T} P-P B_{0} R^{-1} B_{0}^{T} P \\
& +\gamma^{2} P D D^{T} P+\frac{1}{\gamma^{2}} E_{a}^{T} E_{a} \\
& +\zeta^{2} P B_{0} R^{-1} E_{b}^{T} E_{b} R^{-1} B_{0}^{T} P+\frac{1}{\zeta^{2}} P D^{T} D P \leq-Q,
\end{aligned}
$$


where $\rho$ is a prescribed attenuation level, $Q=Q^{T}>0$ is a prescribed weighting matrix, and $R=R^{T}>0$ for some positive gains, then the control law is provided by (15), where $\tau_{0}$ is a fuzzy logic system (16) and $u$ is a robust control law that can be designed as

$$
u=u_{h}+u_{s}
$$

where

$$
\begin{gathered}
u_{h}=-M_{0}(q) R^{-1} B_{0}^{T} P x, \\
u_{s}=-\widehat{\psi} \operatorname{sgn}\left(B_{0}^{T} P x\right), \\
\dot{\hat{\psi}}=\eta B_{0}^{T} P x \operatorname{sgn}\left(B_{0}^{T} P x\right),
\end{gathered}
$$

where $\widehat{\psi}$ is the estimated value of the uncertain term bound $\psi$.

Guarantee that (i) all the variables of the closed-loop system are bounded and (ii) the following $H_{\infty}$ tracking performance is achieved:

$$
\int_{0}^{T}\|x(t)\|_{Q}^{2} d t \leq \rho^{2} \int_{0}^{T}\left\|\tau_{d}\right\|^{2} d t+\beta,
$$

and $\|\theta\| \leq M_{\theta}$. The updating laws of FLS weights are updated by

$$
\dot{\hat{\theta}}=-\Gamma \phi(q, \dot{q}) x^{T} P B_{0} M_{0}^{-1}(q)
$$

where $\Gamma$ is a positive constant adaptation gain matrix.

Proof. Let us select a Lyapunov function candidate:

$$
V=\frac{1}{2} x^{T} P x+\frac{1}{2} \operatorname{tr}\left(\widetilde{\theta}^{T} \Gamma^{-1} \widetilde{\theta}\right)+\frac{1}{2 \eta} \widetilde{\psi}^{2},
$$

where the estimation error is defined as $\widetilde{\psi}=\psi-\widehat{\psi}$. Differentiating the above equation yields

$$
\begin{aligned}
\dot{V}= & \frac{1}{2} \dot{x}^{T} P x+\frac{1}{2} x^{T} P \dot{x}+\operatorname{tr}\left(\widetilde{\theta}^{T} \Gamma^{-1} \dot{\tilde{\theta}}\right)+\frac{1}{\eta} \tilde{\psi} \dot{\tilde{\psi}} \\
= & \frac{1}{2}\left[A x+B\left(u+\tau_{d}+\varepsilon+\widetilde{\theta}^{T} \phi(\cdot)+\omega\right)\right]^{T} P x \\
& +\frac{1}{2} x^{T} P\left[A x+B\left(u+\tau_{d}+\varepsilon+\widetilde{\theta}^{T} \phi(\cdot)+\omega\right)\right] \\
& +\operatorname{tr}\left(\widetilde{\theta}^{T} \Gamma^{-1} \dot{\tilde{\theta}}\right)+\frac{1}{\eta} \widetilde{\psi} \dot{\tilde{\psi}} \\
= & \frac{1}{2} x^{T}\left(P A_{0}+A_{0}^{T} P\right) x+x^{T} P D F E_{a} x \\
& +\left(u+\varepsilon+\widetilde{\theta}^{T} \phi(\cdot)+\omega\right)^{T} B^{T} P x \\
& +\tau_{d} B^{T} P x+\operatorname{tr}\left(\widetilde{\theta}^{T} \Gamma^{-1} \dot{\tilde{\theta}}\right)+\frac{1}{\eta} \tilde{\psi} \dot{\tilde{\psi}} .
\end{aligned}
$$

From (21)-(23) and considering the matrix inequality (27), we obtain

$$
\begin{gathered}
\dot{V} \leq\left(u+\varepsilon+\widetilde{\theta}^{T} \phi(\cdot)+\omega\right)^{T} B^{T} P x+\tau_{d} B^{T} P x \\
+\operatorname{tr}\left(\widetilde{\theta}^{T} \Gamma^{-1} \dot{\tilde{\theta}}\right)+\frac{1}{\eta} \widetilde{\psi} \dot{\widetilde{\psi}}+x^{T} P D F E_{a} x \\
-\frac{1}{2} x^{T}\left\{\frac{1}{\rho^{2}} P \bar{B} \bar{B}^{T} P+Q-P B_{0} R^{-1} B_{0}^{T} P+\gamma^{2} P D D^{T} P\right. \\
+\frac{1}{\gamma^{2}} E_{a}^{T} E_{a}+\eta^{2} P B_{0} R^{-1} E_{b}^{T} E_{b} R^{-1} B_{0}^{T} P \\
\left.+\frac{1}{\eta^{2}} P D^{T} D P\right\} x .
\end{gathered}
$$

Considering $u_{s}$ in the robust controller equation (30) and the fact $\dot{\tilde{\theta}}=-\dot{\hat{\theta}}$, we obtain

$$
\operatorname{tr}\left(\widetilde{\theta}^{T} \Gamma^{-1} \dot{\tilde{\theta}}\right)+\left(u_{s}+\varepsilon+\widetilde{\theta}^{T} \phi(\cdot)+\omega\right)^{T} B^{T} P x+\frac{1}{\eta} \tilde{\psi} \dot{\tilde{\psi}} \leq 0 .
$$

Substituting (37) into (36) yields

$$
\begin{aligned}
\dot{V} \leq & -\frac{1}{2} x^{T}\left\{\frac{1}{\rho^{2}} P \bar{B} \bar{B}^{T} P+Q-P B_{0} R^{-1} B_{0}^{T} P\right. \\
& +\gamma^{2} P D D^{T} P+\frac{1}{\gamma^{2}} E_{a}^{T} E_{a} \\
& \left.+\eta^{2} P B_{0} R^{-1} E_{b}^{T} E_{b} R^{-1} B_{0}^{T} P+\frac{1}{\eta^{2}} P D^{T} D P\right\} x \\
& +x^{T} P D F E_{a} x+u_{h}^{T} B^{T} P x+\tau_{d} B^{T} P x \\
= & -\frac{1}{2 \rho^{2}} x^{T} P\left(\bar{B} \bar{B}^{T}-B B^{T}\right) P x \\
& -\frac{1}{2}\left(\frac{1}{\rho} B^{T} P x-\rho \tau_{d}\right)^{T}\left(\frac{1}{\rho} B^{T} P x-\rho \tau_{d}\right) \\
& -\frac{1}{2}\left(\gamma D^{T} P x-\frac{1}{\gamma} F E_{a} x\right)^{T}\left(\gamma D^{T} P x-\frac{1}{\gamma} F E_{a} x\right) \\
& -\frac{1}{2 \gamma^{2}} x^{T} E_{a}^{T}\left(I_{n \times n}-F^{T} F\right) E_{a} x+\frac{1}{2} \rho^{2} \tau_{d}^{T} \tau_{d}-\frac{1}{2} x^{T} Q x \\
& -\frac{1}{2}\left(\eta F E_{b} R^{-1} B_{0}^{T} P x+\frac{1}{\eta} D^{T} P x\right)^{T} \\
& +\left(\eta F E_{b} R^{-1} B_{0}^{T} P x+\frac{1}{\eta} D^{T} P x\right) \\
& +B_{0} R^{-1} E_{b}^{T}\left(I_{n \times n}-F^{T} F\right) E_{b} R^{-1} B_{0}^{T} P x \\
& \\
& =\frac{1}{2} P(38)
\end{aligned}
$$

Then,

$$
\dot{V} \leq \frac{1}{2} \rho^{2} \tau_{d}^{T} \tau_{d}-\frac{1}{2} x^{T} Q x .
$$




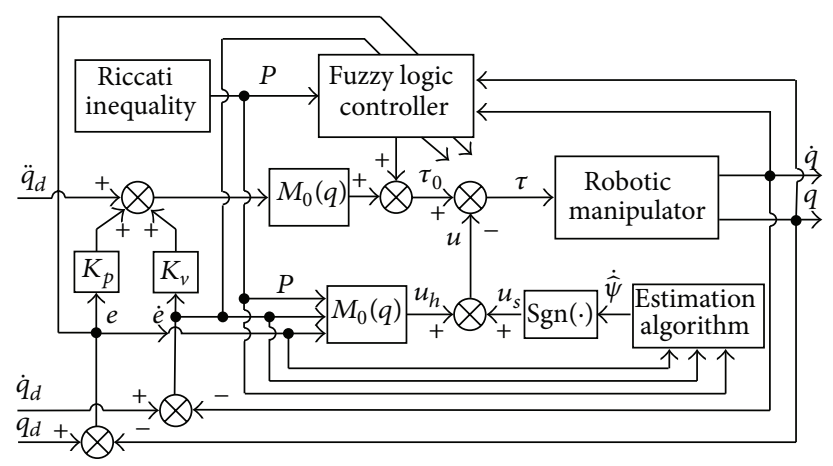

FIgURE 2: The architecture of closed-loop system.

Integrating the above inequality from $t=0$ to $t=T$ yields

$$
V(T)-V(0) \leq \frac{1}{2} \rho^{2} \int_{0}^{T} \tau_{d}^{T} \tau_{d} d t-\frac{1}{2} \int_{0}^{T} x^{T} Q x d t
$$

Since $V(T) \geq 0$, the above inequality leads to

$$
\begin{aligned}
\int_{0}^{T}\|x(t)\|_{\mathrm{Q}}^{2} d t \leq & x^{T}(0) P x(0) \\
& +\operatorname{tr}\left(\widetilde{\theta}^{T}(0) \Gamma^{-1} \widetilde{\theta}(0)\right)+\rho^{2} \int_{0}^{T}\left\|\tau_{d}\right\|^{2} d t .
\end{aligned}
$$

Defining $\beta=x^{T}(0) P x(0)+\operatorname{tr}\left(\widetilde{\theta}^{T}(0) \Gamma^{-1} \widetilde{\theta}(0)\right)$, then the $H_{\infty}$ performance is achieved. Since $\tau_{d} \in L_{2}[0,+\infty)$, there is a finite constant $M_{d}>0$ such that $\int_{0}^{T}\left\|\tau_{d}\right\|^{2} d t \leq M_{d}$; then, we have

$$
\|x\| \leq \sqrt{\frac{\beta+\rho^{2} M_{d}}{\lambda_{\min }(Q)}} .
$$

It can be concluded that all the signals of the closed-loop system are bounded.

According to the above analysis, the architecture of closed-loop system is shown in Figure 2.

Remark 12. Noting that the inputs of designed controller include velocity signals $\dot{q}$, here it is assumed that the velocities are measurable. Compared with $[5,16]$, the acceleration signals $\ddot{q}$ are no longer needed to be measurable.

Remark 13. Since the controller equation (30) contains the sign function, direct application of such control signals to the robotic system (6) may result in chattering caused by the signal discontinuity. To overcome this problem, the control law is smoothed out within a thin boundary layer $\Phi_{i}[18]$ by replacing the sign function by a saturation function defined as

$$
\operatorname{sat}\left(\frac{\left(B_{0}^{T} P x\right)_{i}}{\Phi_{i}}\right)= \begin{cases}\operatorname{sgn}\left(\frac{\left(B_{0}^{T} P x\right)_{i}}{\Phi_{i}}\right), & \left\|\frac{\left(B_{0}^{T} P x\right)_{i}}{\Phi_{i}}\right\|>1, \\ \frac{\left(B_{0}^{T} P x\right)_{i}}{\Phi_{i}}, & \left\|\frac{\left(B_{0}^{T} P x\right)_{i}}{\Phi_{i}}\right\| \leq 1 .\end{cases}
$$

Remark 14. The above stability result is achieved under the assumption that all the parameter vectors are within the constraint sets or on the boundaries of the constraint set but moving their interior $\left(\|\theta\| \leq M_{\theta}\right)$. To guarantee that the parameters are bounded, the adaptive laws (33) can be modified by using the projection algorithm [11] as follows:

$$
\dot{\hat{\theta}}=\left\{\begin{array}{l}
-\Gamma \phi(\cdot) x^{T} P B_{0} M_{0}^{-1}(q), \\
\quad \text { if }\|\hat{\theta}\|<M_{\theta} \text { or } \\
\quad\left(\|\hat{\theta}\|=M_{\theta}, x^{T} P B_{0} M_{0}^{-1}(q) \hat{\theta}^{T} \phi(\cdot) \geq 0\right), \\
\operatorname{Pr}(\cdot), \\
\quad \text { if }\left(\|\hat{\theta}\|=M_{\theta}, x^{T} P B_{0} M_{0}^{-1}(q) \hat{\theta}^{T} \phi(\cdot)<0\right),
\end{array}\right.
$$

where the projection operator $\operatorname{Pr}(\cdot)$ is defined as

$$
\operatorname{Pr}(\cdot)=-\Gamma \phi(\cdot) x^{T} P B_{0} M_{0}^{-1}(q)+\frac{\Gamma x^{T} P B_{0} M_{0}^{-1}(q) \widehat{\theta}^{T} \phi(\cdot)}{\|\hat{\theta}\|^{2}}
$$


TABLE 1: Simulation parameters.

\begin{tabular}{lcc}
\hline & Nominal values & Actual values \\
\hline$m_{1}(\mathrm{~kg})$ & 1 & 2 \\
$m_{2}(\mathrm{~kg})$ & 1 & $1+\sin 0.5 t$ \\
$l_{1}(\mathrm{~m})$ & 1.1 & 1.2 \\
$l_{2}(\mathrm{~m})$ & 0.8 & 1.2 \\
\hline
\end{tabular}

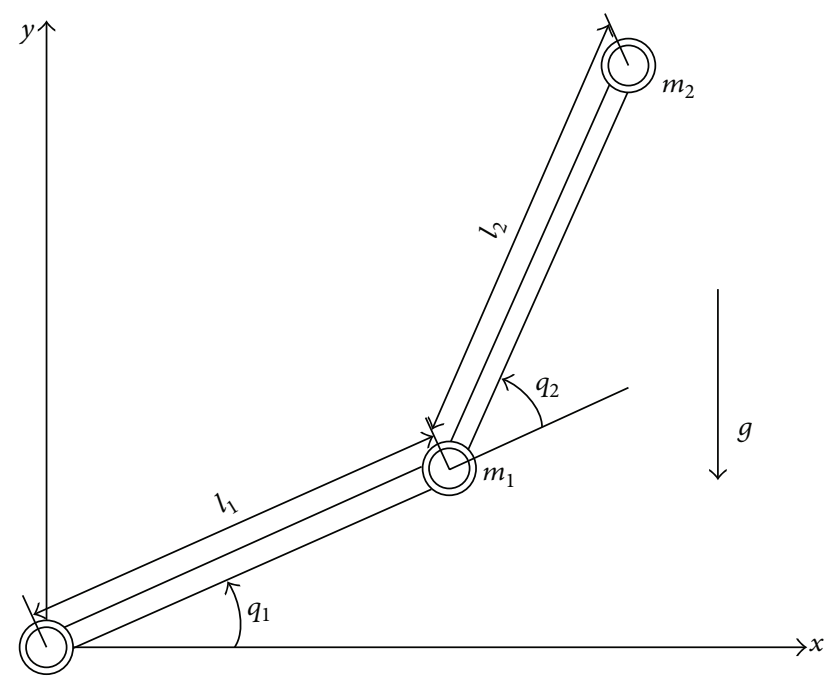

FIgURE 3: Diagram of a two-link robot manipulator.

\section{Simulation Example}

To verify the theoretical results, simulations are carried out in two degrees of freedom robot manipulator as shown in Figure 3 described by $[6,17]$

$$
\begin{gathered}
M(q) \\
=\left[\begin{array}{cc}
m_{1} l_{1}^{2}+m_{2}\left(l_{1}^{2}+l_{2}^{2}+2 l_{1} l_{2} c_{2}\right) & m_{2} l_{2}^{2}+m_{2} l_{1} l_{2} c_{2} \\
m_{2} l_{2}^{2}+m_{2} l_{1} l_{2} c_{2} & m_{2} l_{2}^{2}
\end{array}\right], \\
C(q, \dot{q})=\left[\begin{array}{cc}
-2 m_{2} l_{1} l_{2} s_{2} \dot{q}_{2} & m_{2} l_{1} l_{2} s_{2} \dot{q}_{2} \\
m_{2} l_{1} l_{2} s_{2} \dot{q}_{1} & 0
\end{array}\right], \\
G(q)=\left[\begin{array}{cc}
m_{2} l_{2} g c_{12}+\left(m_{1}+m_{2}\right) & l_{1} g c_{1} \\
m_{2} l_{2} g c_{12}
\end{array}\right],
\end{gathered}
$$

where $m_{1}$ and $m_{2}$ are the mass of link 1 and link 2, respectively; $l_{1}$ and $l_{2}$ are the length of link 1 and link 2 , respectively; $s_{i}$ denotes $\sin q_{i} ; c_{i}$ denotes $\cos q_{i}$; and $c_{i j}$ denotes $\cos \left(q_{i}+q_{j}\right)$ for $i=1,2$ and $j=1,2 . g$ is acceleration of gravity.

The robotic manipulator parameters used for the simulation are shown in Table 1, where the nominal values are used to calculate the controller functions to design the robust adaptive controller and the actual values are used to test the robustness of the controller. Furthermore, the external disturbances are assumed to be $\tau_{d}=\left[\begin{array}{lll}-5 \cos (5 t) & -5 \sin (5 t)\end{array}\right]^{T}$. The simulation sampling steps length is given by $0.01 \mathrm{~s}$.
Select controller gains $K_{p}=\operatorname{diag}(50,50)$ and $K_{v}=$ $\operatorname{diag}(20,20)$ such that

$$
A=\left[\begin{array}{cc}
0_{2 \times 2} & I_{2 \times 2} \\
-10 I_{2 \times 2} & -5 I_{2 \times 2}
\end{array}\right]
$$

is a Hurwitz matrix. Choose proper parameters $Q=\operatorname{diag}(15$, $15,15,15), R=\operatorname{diag}(10,10,10,10), \rho=0.1, \gamma=1, \eta=1, D=$ $\operatorname{diag}(0.01,0.01,0.01,0.01), E_{a}=\operatorname{diag}(0.01,0.01,0.01,0.01)$, and $E_{b}=\operatorname{diag}(0,0,0.01,0.01)$, then slove $P$ from matrix inequalities (23):

$$
P=\left[\begin{array}{cc}
30.1642 I_{2 \times 2} & 0.0653 I_{2 \times 2} \\
0.0653 I_{2 \times 2} & 0.1719 I_{2 \times 2}
\end{array}\right] .
$$

The inputs of fuzzy logic system are taken as $q_{1}, q_{2}, \dot{q}_{1}$, $\dot{q}_{2}, q_{d 1}$, and $q_{d 2}$, and for each input vector, six membership functions are defined as

$$
\begin{aligned}
& \mu_{F_{i}^{1}}\left(x_{i}\right)=\frac{1}{1+\exp \left\{5\left(x_{i}+2\right)\right\}}, \\
& \mu_{F_{i}^{2}}\left(x_{i}\right)=\exp \left\{-\left(x_{i}+1.5\right)^{2}\right\}, \\
& \mu_{F_{i}^{3}}\left(x_{i}\right)=\exp \left\{-\left(x_{i}+0.5\right)^{2}\right\}, \\
& \mu_{F_{i}^{4}}\left(x_{i}\right)=\exp \left\{-\left(x_{i}-0.5\right)^{2}\right\}, \\
& \mu_{F_{i}^{5}}\left(x_{i}\right)=\exp \left\{-\left(x_{i}-1.5\right)^{2}\right\}, \\
& \mu_{F_{i}^{6}}\left(x_{i}\right)= \frac{1}{1+\exp \left\{-5\left(x_{i}-2\right)\right\}}, \\
& i=1,2 .
\end{aligned}
$$

The gain matrix is selected as $\Gamma=\operatorname{diag}(0.1,0.1,0.1,0.1)$ in updating law (29). The initial values of weight parameters $\theta(0)$ are set equal to zero matrix. The initial conditions are $q_{1}(0)=0.5 \mathrm{rad}, q_{2}(0)=-0.5 \mathrm{rad}, \dot{q}_{1}(0)=\dot{q}_{2}(0)=0 \mathrm{rad} / \mathrm{s}^{2}$, and $\widehat{\psi}_{1}(0)=\widehat{\psi}_{2}(0)=0.2$. The desired trajectories to be tracked are $q_{d 1}=\sin (t)$ and $q_{d 2}=\cos (t)$.

For comparison, the conventional CTC equation (12) under the same conditions is also demonstrated. The gains are chosen as $K_{p}=\operatorname{diag}[50,50]$ and $K_{v}=\operatorname{diag}[20,20]$. Figures 4 and 5 show the tracking results of $q_{1}$ and $q_{2}$ with CTC and the proposed FLS-based robust tracking control method, Figures 6 and 7 show the tracking errors $e_{1}$ and $e_{2}$, and Figures 8 and 9 show the control inputs $u_{1}$ and $u_{2}$, respectively. Figures 10 and 11 show the weights $\theta_{1}$ and $\theta_{2}$ of fuzzy logic system. It can be seen that the CTC controller cannot drive the joints to reach the desired positions and steadystate tracking error exists, while the tracking errors go to small values by the proposed method after some transients, which are caused by the initial choice of the consequent parameters. However, the tracking error decreases quickly since the online learning of fuzzy logic system and the effect of uncertainties are successfully compensated by the robust control term. The simulation results thus demonstrate that the proposed FLS-based robust tracking control method can 


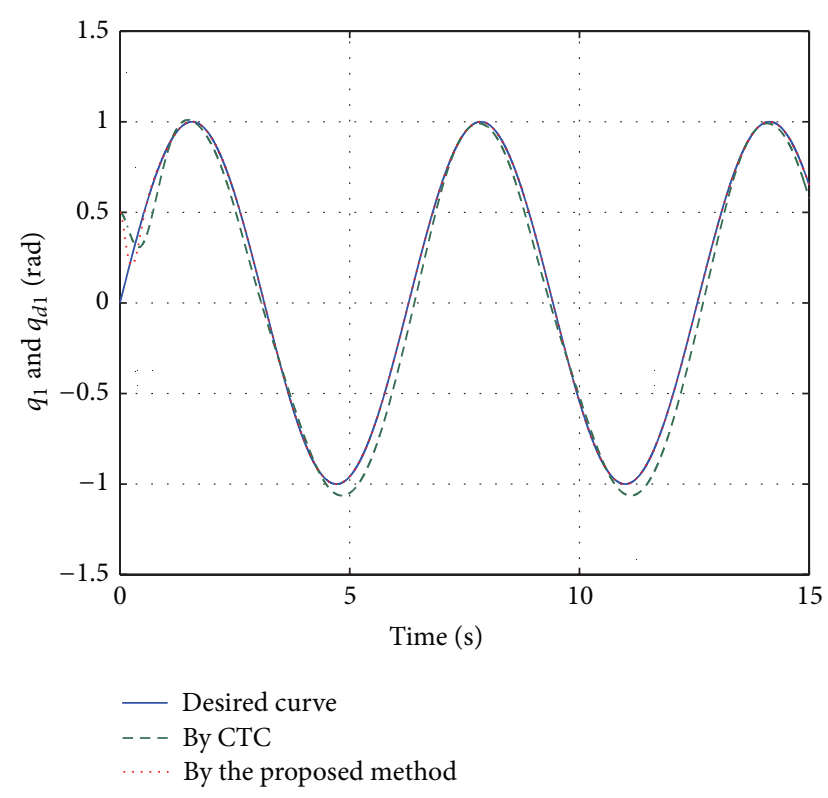

FIGURE 4: Trajectory tracking of $q_{1}$.

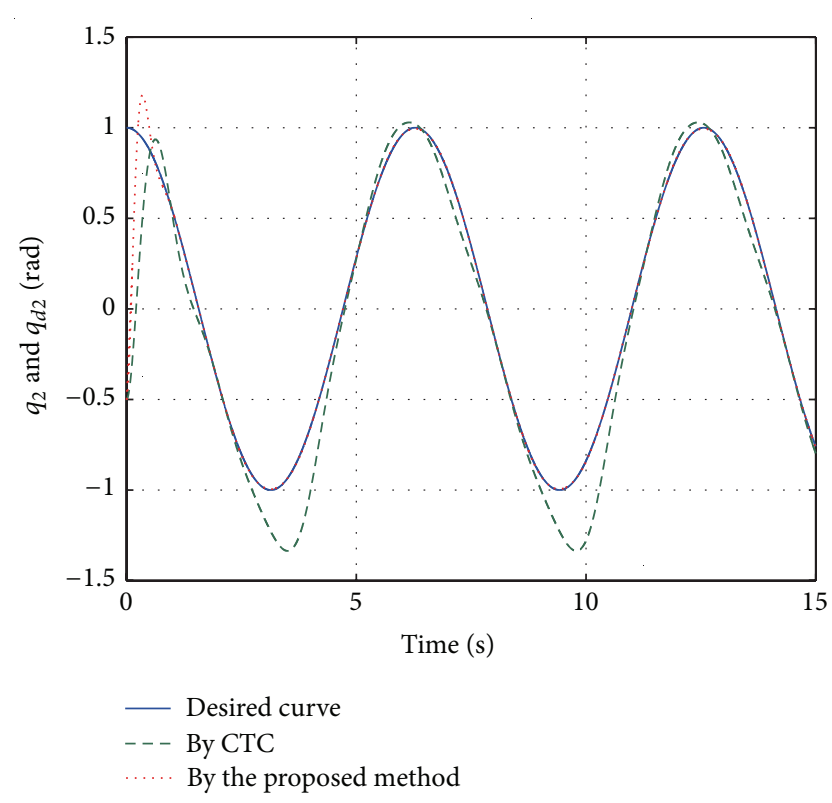

Figure 5: Trajectory tracking of $q_{2}$.

effectively control the rigid robotic manipulator with uncertainties.

To quantify the control performance, the root-mean square average of tracking error (based on the $L_{2}$ norm of the tracking errors $e$ ) is given as follows $[6,16]$ :

$$
L_{2}(e)=\sqrt{\frac{1}{T} \int_{0}^{T} e^{T} e d t},
$$

where $T$ represents the total simulation time. Table 2 shows the $L_{2}$ error norms for CTC method, adaptive robust quadratic stabilization tracking control (ARQSTC) method [6],

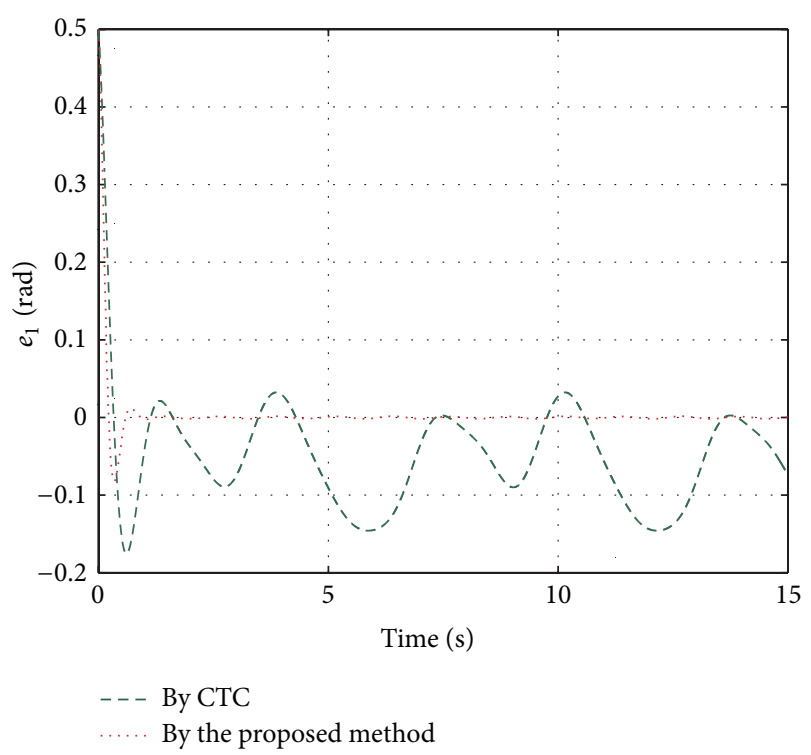

FIgURE 6: Tracking error $e_{1}$.

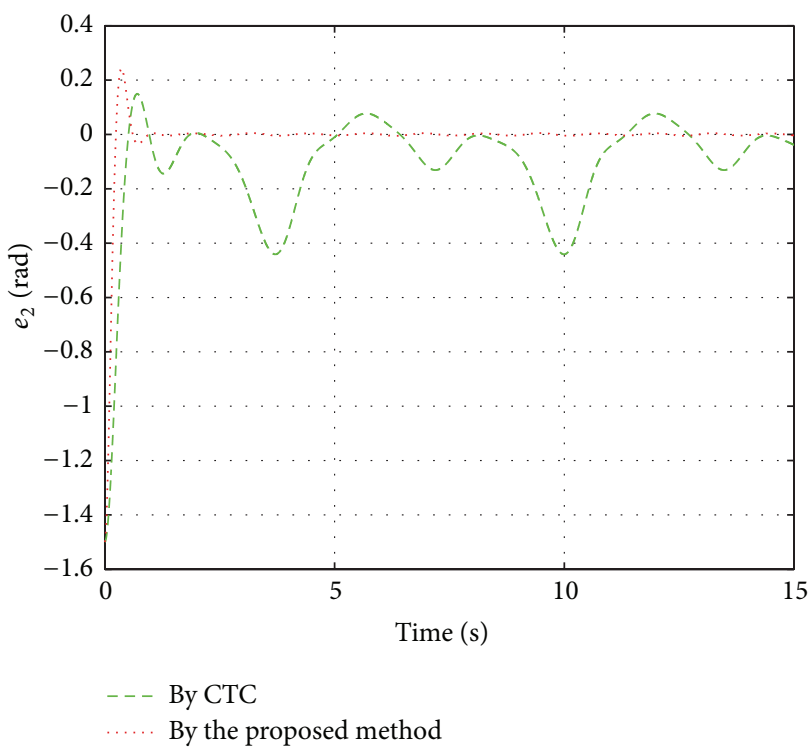

Figure 7: Tracking error $e_{2}$.

TABLE 2: $L_{2}$ norm for tracking errors.

\begin{tabular}{lcc}
\hline Controller & $L_{2}\left(e_{1}\right)$ & $L_{2}\left(e_{2}\right)$ \\
\hline CTC & 5.8827 & 11.0905 \\
ARQSTC & 3.7336 & 7.4509 \\
NNRHC & 1.1345 & 1.5158 \\
Proposed & 1.9438 & 3.7406 \\
\hline
\end{tabular}

neural network-based robust $H_{\infty}$ control (NNRHC) method [16], and the proposed FLS-based robust tracking control method. Note that a smaller $L_{2}$ norm represents a better performance.

From Table 2, the proposed control method shows the smaller tracking error norm in comparison to the CTC and 


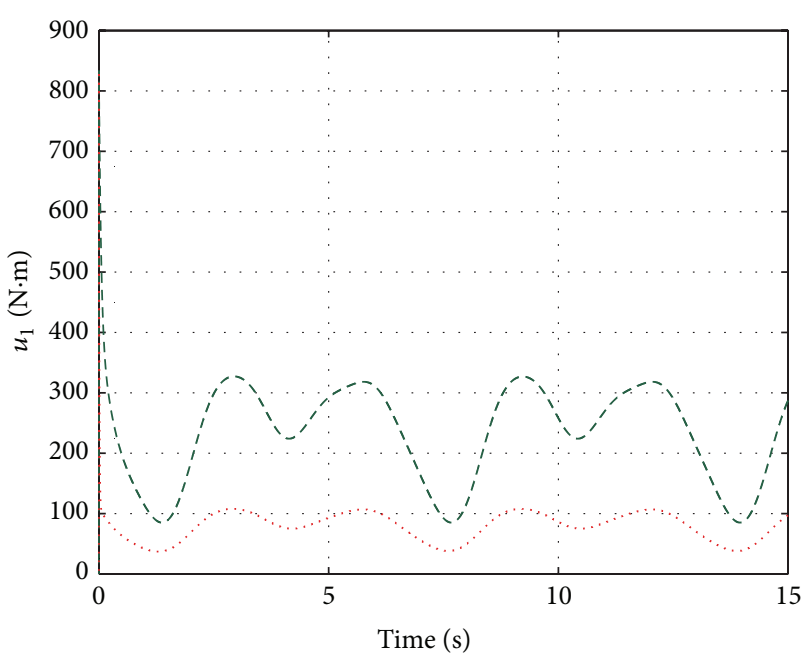

- - By CTC

By the proposed method

FIgURE 8: Control input $u_{1}$.

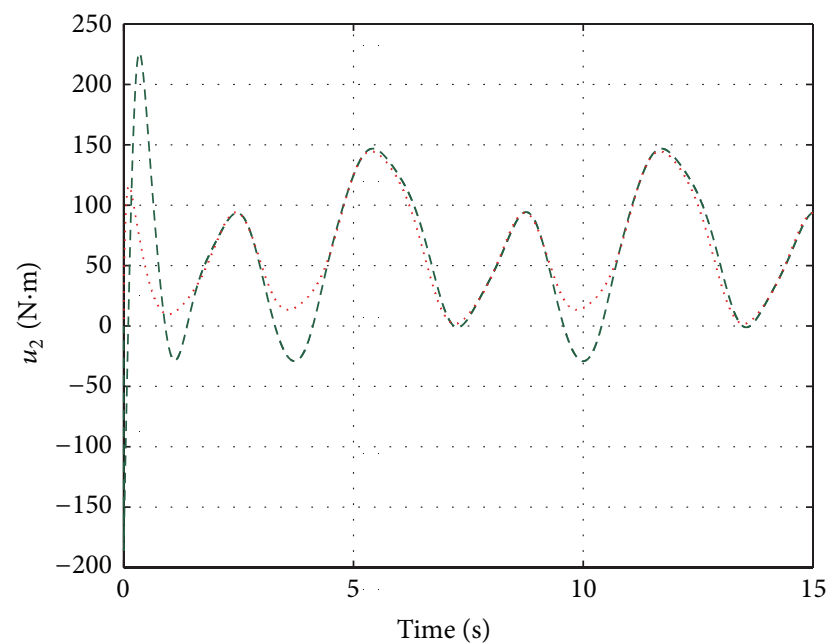

By CTC

By the proposed method

FIgURE 9: Control input $u_{2}$.

ARQSTC methods and the slight larger tracking error norm in comparison to the NNRHC method. It should be noted that the system nominal model was assumed to be known exactly and the acceleration signal $\ddot{q}$ was assumed to be measurable, which were both used to implement the NNRHC methods, while the system nominal model and acceleration signal were not included in the proposed controller. In general, more system information used in controller design leads to a better tracking performance.

\section{Conclusions}

This paper presents a robust tracking control scheme for robotic manipulator with mathematical derivations of global

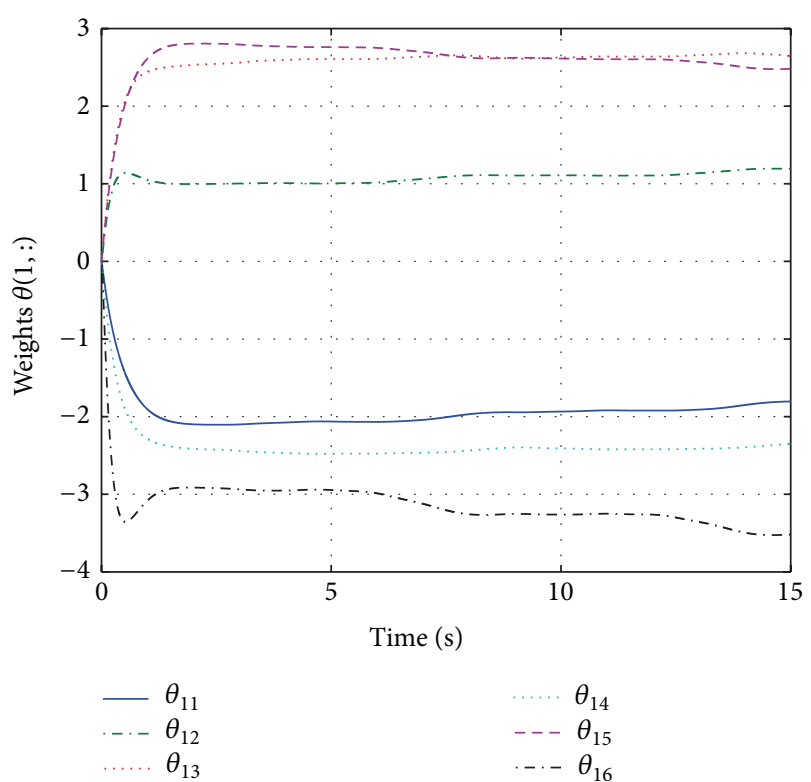

Figure 10: Weights $\theta_{1}$ of fuzzy logic system.

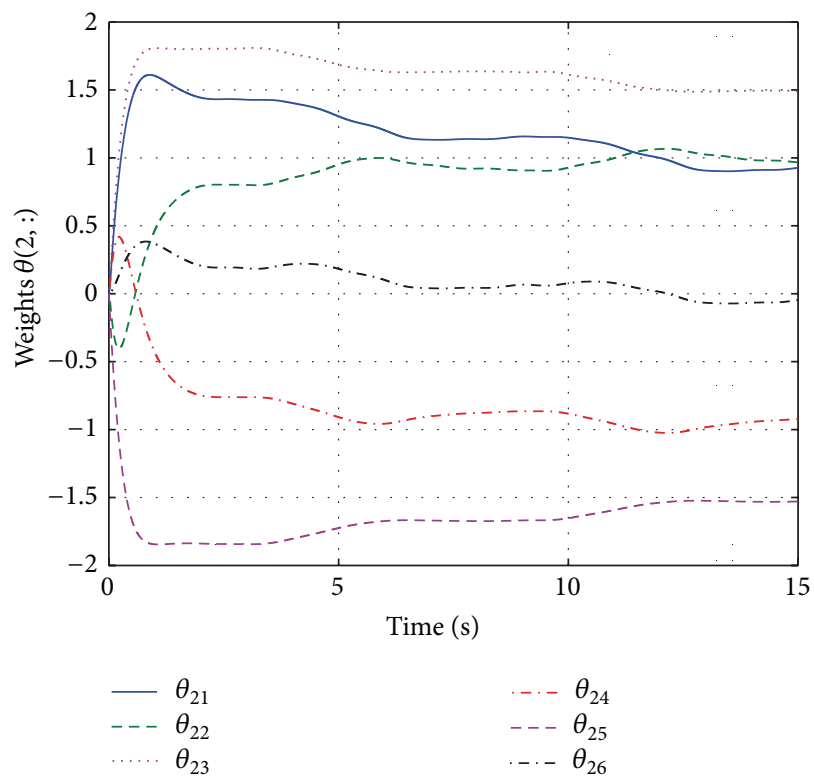

Figure 11: Weights $\theta_{2}$ of fuzzy logic system.

stability. The idea is to combine an FLS controller with a robust compensator. Here, we assumed that the nominal parameters of robotic manipulator are unknown except the nominal inertia matrix. The FLS is employed to implement the framework of CTC method which acts as the main controller, and the robust compensator is used to handle system uncertainties and external disturbances. In addition, an $H_{\infty}$ controller is used to achieve a certain tracking performance. The intelligent hybrid control method demonstrated robust and effective control performance on robotic manipulator having uncertainties with good disturbance rejection. 


\section{Conflict of Interests}

The authors declare that there is no conflict of interests regarding the publication of this paper.

\section{Acknowledgments}

The authors would like to acknowledge the funding received from the China Postdoctoral Science Foundation (nos. 2014T70685 and 2013M541992), the Specialized Research Fund for the Doctoral Program of Higher Education of China (no. 20124101120001), and Key Project for Science and Technology of the Education Department of Henan Province (no. 14A413009) to conduct this research investigation.

\section{References}

[1] W. J. Book, "Modeling, design, and control of flexible manipulator arms: a tutorial review," in Proceedings of the 29th IEEE Conference on Decision and Control, pp. 500-506, IEEE, Honolulu, Hawaii, USA, December 1990.

[2] F. L. Lewis, S. Jagannathan, and A. Yesildirek, Neural Network Control of Robot Manipulators and Nonlinear Systems, SIAM Press, Philadelphia, Pa, USA, 2002.

[3] J. Y. S. Luh, "Conventional controller design for industrial robots-a tutorial," IEEE Transactions on Systems, Man and Cybernetics, vol. 13, no. 3, pp. 298-316, 1983.

[4] R. H. Middleton and G. C. Goodwin, "Adaptive computed torque control for rigid link manipulations," Systems and Control Letters, vol. 10, no. 1, pp. 9-16, 1988.

[5] Z. Song, J. Yi, D. Zhao, and X. Li, "A computed torque controller for uncertain robotic manipulator systems: fuzzy approach," Fuzzy Sets and Systems, vol. 154, no. 2, pp. 208-226, 2005.

[6] J. Peng and Y. Liu, "Adaptive robust quadratic stabilization tracking control for robotic system with uncertainties and external disturbances," Journal of Control Science and Engineering, vol. 2014, Article ID 715250, 10 pages, 2014.

[7] K. S. Yeung and Y. P. Chen, "A new controller design for manipulators using the theory of variable structure systems," IEEE Transactions on Automatic Control, vol. 33, no. 2, pp. 200206, 1988.

[8] M. Chen, C. Jiang, and Q. Wu, "Backstepping control for a class of uncertain nonlinear systems with neural network," International Journal of Nonlinear Science, vol. 3, no. 2, pp. 137-143, 2007.

[9] O. Kaynak, K. Erbatur, and M. Ertugrul, "The fusion of computationally intelligent methodologies and sliding-mode control-a survey," IEEE Transactions on Industrial Electronics, vol. 48, no. 1, pp. 4-17, 2001.

[10] F. C. Sun, Z. Q. Sun, and G. Feng, "An adaptive fuzzy controller based on sliding mode for robot manipulators," IEEE Transactions on Systems, Man, and Cybernetics, Part B: Cybernetics, vol. 29, no. 5, pp. 661-667, 1999.

[11] Y. C. Hsu, G. Chen, and H. X. Li, "A fuzzy adaptive variable structure controller with applications to robot manipulators," IEEE Transactions on Systems, Man, and Cybernetics, Part B: Cybernetics, vol. 31, no. 3, pp. 331-340, 2001.

[12] H. Hu and P.-Y. Woo, "Fuzzy supervisory sliding-mode and neural-network control for robotic manipulators," IEEE Transactions on Industrial Electronics, vol. 53, no. 3, pp. 929-940, 2006.
[13] F. L. Lewis, K. Liu, and A. Yesildirek, "Neural net robot controller with guaranteed tracking performance," IEEE Transactions on Neural Networks, vol. 6, no. 3, pp. 703-715, 1995.

[14] F. L. Lewis, A. Yeşildirek, and K. Liu, "Multilayer neural-net robot controller with guaranteed tracking performance," IEEE Transactions on Neural Networks, vol. 7, no. 2, pp. 388-399, 1996.

[15] Y.-C. Chang, "Neural network-based $H_{\infty}$ tracking control for robotic systems," IEE Proceedings-Control Theory and Applications, vol. 147, no. 3, pp. 303-311, 2000.

[16] Y. Zuo, Y. Wang, X. Liu et al., "Neural network robust $H_{\infty}$ tracking control strategy for robot manipulators," Applied Mathematical Modelling, vol. 34, no. 7, pp. 1823-1838, 1838.

[17] J. Peng, J. Wang, and Y. Wang, "Neural network based robust hybrid control for robotic system: an $H_{\mathrm{\infty}}$ approach," Nonlinear Dynamics, vol. 65, no. 4, pp. 421-431, 2011.

[18] J. Peng and R. Dubay, "Nonlinear inversion-based control with adaptive neural network compensation for uncertain MIMO systems," Expert Systems with Applications, vol. 39, no. 9, pp. 8162-8171, 2012.

[19] E.-J. Kim, K. Seki, and M. Iwasaki, "Motion control of industrial robots by considering serial two-link robot arm model with joint nonlinearities," Journal of Mechanical Science and Technology, vol. 28, no. 4, pp. 1519-1527, 2014.

[20] Y. Chen, G. Ma, S. Lin, and J. Gao, "Adaptive fuzzy computedtorque control for robot manipulator with uncertain dynamics," International Journal of Advanced Robotic Systems, vol. 9, pp. 19, 2012.

[21] J. Peng, Y. Liu, and J. Wang, "Fuzzy adaptive output feedback control for robotic systems based on fuzzy adaptive observer," Nonlinear Dynamics, vol. 78, no. 2, pp. 789-801, 2014.

[22] J. J. E. Slotine and W. Li, Applied Nonlinear Control, PrenticeHall, Englewood Cliffs, NJ, USA, 1991. 

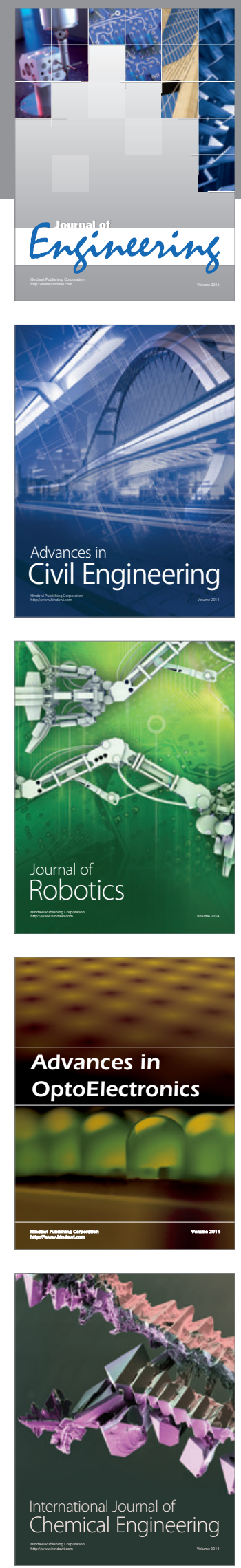

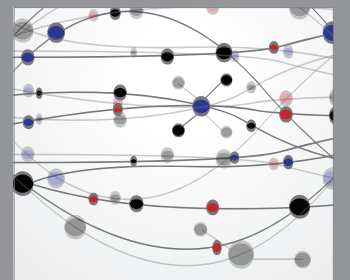

The Scientific World Journal
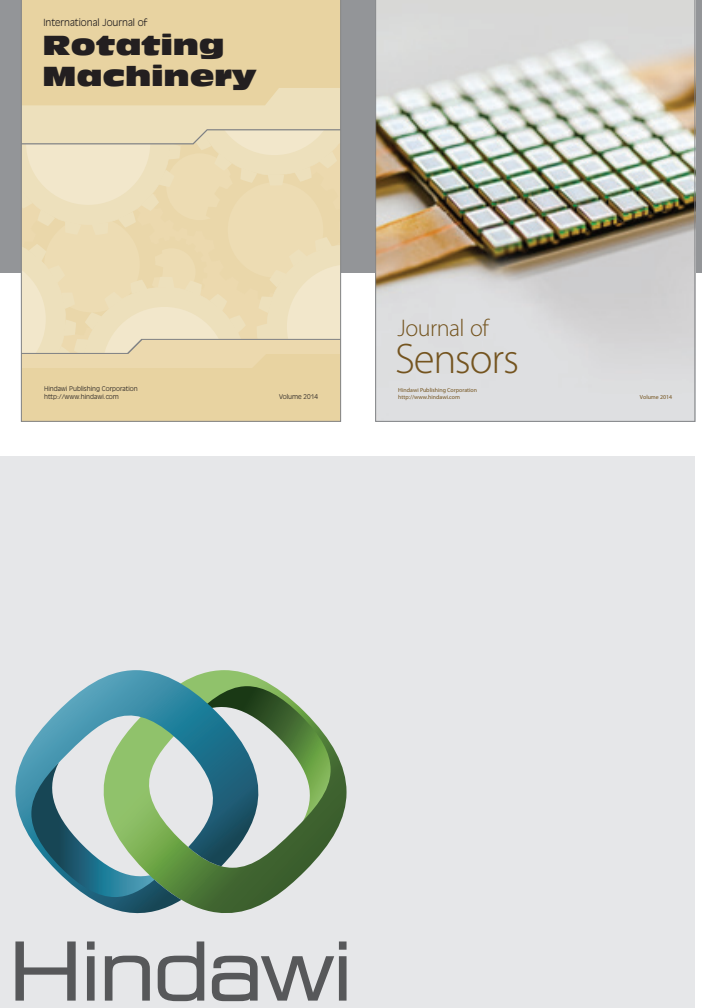

Submit your manuscripts at http://www.hindawi.com
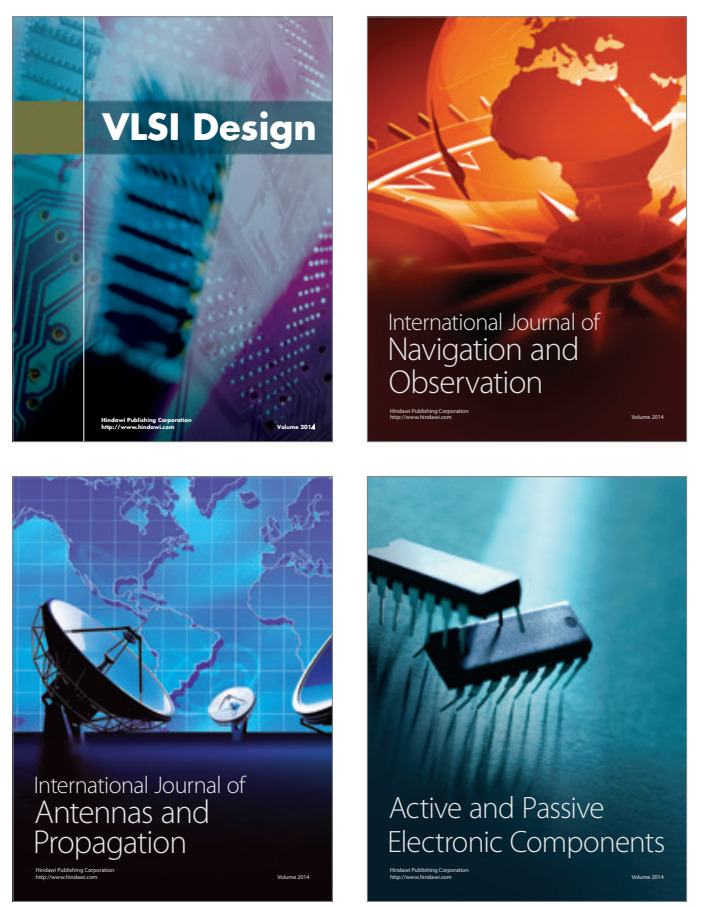
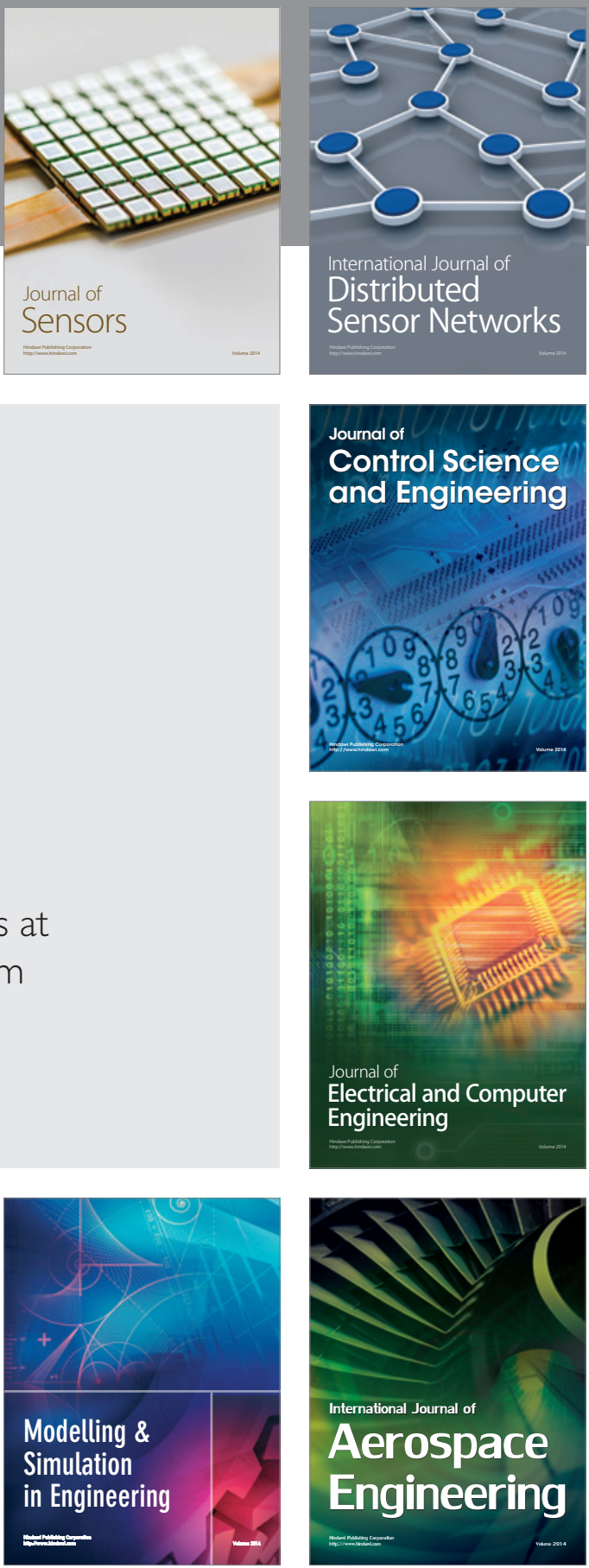

Journal of

Control Science

and Engineering
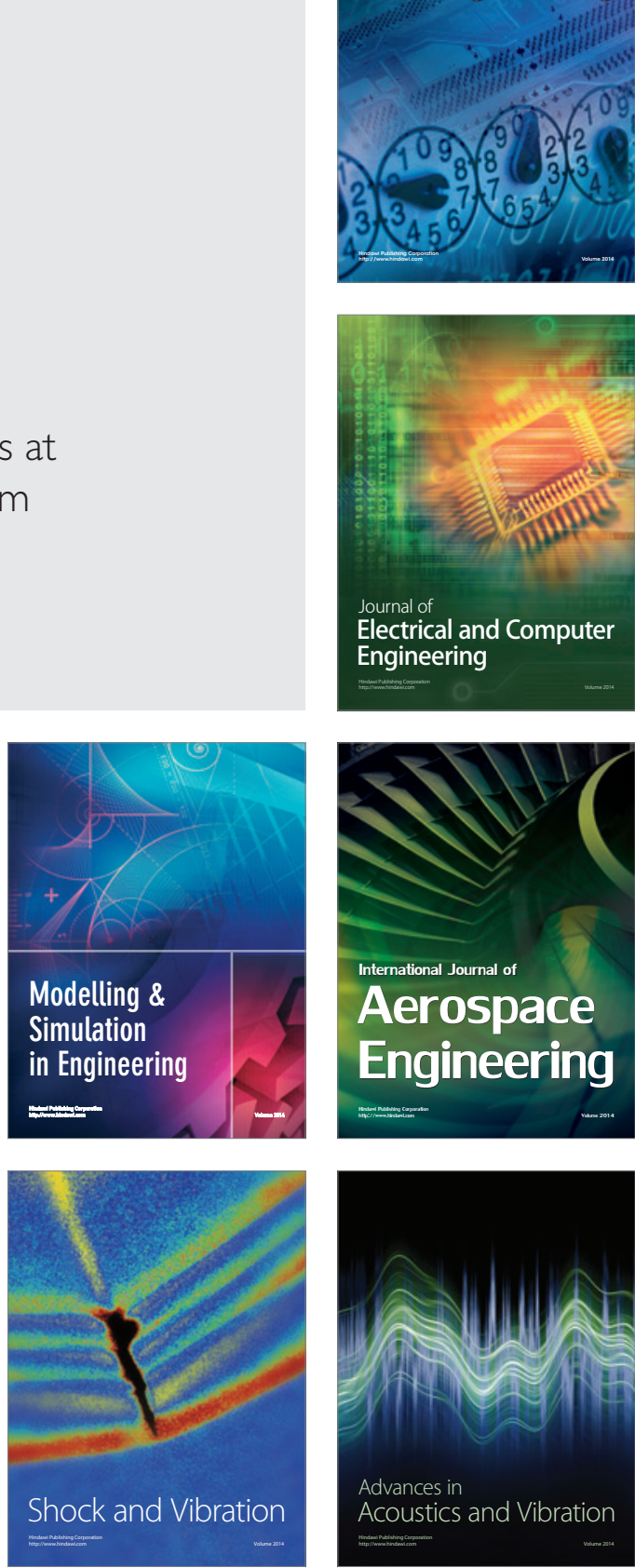